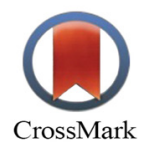

\title{
Hepatitis B Vaccine Acceptability among Nurses: A Theory Based Conceptualization
}

\author{
Mehdi Mirzaei Alavijeh ${ }^{1}$, Masoumeh Vaezi ${ }^{2}$, Farzad Jalilian ${ }^{1, *}$
}

1. Social Development and Health Promotion Research Center, Kermanshah University of Medical Sciences, Kermanshah, Iran

2. Faculty of Medicine, Kateb University, Kabul, Afghanistan

\section{* Corresponding Author:}

Farzad Jalilian, PhD

Social Development \& Health Promotion Research Center, Kermanshah University of Medical Sciences, Kermanshah, Iran Telefax: + 988334216143

Email: f jalilian@yahoo.com

Received: 25 May 2018

Accepted: 10 Sep. 2018

\section{ABSTRACT}

\section{BACKGROUND}

Hepatitis B is the most common occupational disease in health care providers. It can be followed by several complications. The aim of this study was to determine the cognitive determinants of hepatitis B (HB) vaccination Acceptability among nurses based on the Health Belief Model (HBM).

\section{METHODS}

This cross-sectional study was conducted among 330 nurses in educational hospitals of Kermanshah city, during 2016. Samples were randomly selected with the proportional to size among different educational hospitals in Kermanshah. A structured questionnaire was applied for collecting data and data were analyzed using SPSS software version 16 by using bivariate correlations and logistic regression statistical tests.

\section{RESULTS}

The mean age of the respondents was 30.5 years [SD: 6.62]. About, $58.5 \%$ of the participants reported to have completed (three times) vaccination of HB. Female nurses were more likely to be fully vaccinated against $\mathrm{HB}$ than male nurses, with adjusted odds ratio estimate of 2.507 [95\% CI: 1.523-4.125] and those who had family or friends with a history of HB with odds ratio estimate of 3.706 [95\% CI: 1.317-10.425], making these the most influential predictive determinants for full uptake (three time) of $\mathrm{HB}$ vaccination. Among the HBM variables: perceived threat with odds ratio estimate of 1.264 [95\% CI: 1.160-1.376], perceived self-efficacy with odds ratio estimate of 1.179 [95\% CI: 1.020-1.363], and cues to action with odds ratio estimate of 1.335 [95\% CI: 1.015-1.756], were the more influential predictors of full uptake (three time) of HB vaccination.

\section{CONCLUSION}

To development uptake vaccination programs promotion in addition to focusing on male nurses, using the results of the predictive constructs include; perceived threat perceived self-efficacy suggested. Based on our result, it seems that development and implementation of health promotion programs to increase threat about side effect of $\mathrm{HB}$ and self-efficacy regarding $\mathrm{HB}$ vaccination uptake may be usefulness of the findings to promotion of vaccination of $\mathrm{HB}$.

\section{KEYWORDS:}

Health promotion, Health education, Public health, Behavioral medicine

Please cite this paper as:

Mirzaei Alavijeh M, Vaezi M, Jalilian F. Hepatitis B Vaccine Acceptability among Nurses: A Theory Based Conceptualization. Middle East J Dig Dis 2019;11:45-51. doi: 10.15171/ mejdd.2018.127.

\section{INTRODUCTION}

Hepatitis B (HB) is the cause of many acute and chronic infections, liver cirrhosis, and liver cancer worldwide. ${ }^{1} \mathrm{HB}$ is the most common occupational disease in health care providers and its annual incidence in hospital staff is about two to ten times more than that of the general population. ${ }^{2}$ Vaccination 
is a very appropriate strategy to prevent $\mathrm{HB}$ virus (HBV) infection. ${ }^{3}$ Chronic infection with HB is still one of the most important public health issues in the world despite the presence of effective vaccines and strong antiviral treatments. During 1990-2014 Iran had a 3\% prevalence of $\mathrm{HB}$, according to Mohammadi and colleagues. ${ }^{4}$ In 2014, the $67^{\text {th }}$ World health assembly emphasized the need for monitoring the prevention, diagnosis, and treatment of viral hepatitis. ${ }^{5}$ For this purpose, accurate epidemiological information at the national level is necessary to assess the impact of implemented strategies. ${ }^{6}$ Iran has a moderate prevalence of $\mathrm{HBV} .^{7}$ The prevalence of chronic HB infection is $1.7 \%$. Therefore, with the aim of preventing $\mathrm{HB}$, vaccination of high-risk groups, including health care workers in Iran, began in 1993, but the results of a survey of various $\mathrm{HB}$ vaccination coverage in health care workers showed rates between $52.7 \%$ and $87.2 \%{ }^{8}$ Therefore, considering the full implementation of the $\mathrm{HB}$ vaccination program among health care providers, it is necessary to examine the beliefs associated with performing this health behavior, which can guide specialists in planning vaccination promotion programs. ${ }^{9-11}$

Social-cognitive determinants are among the main causes of behavior change, and these determinants are dependent on factors such as past experiences and sociocultural processes. ${ }^{11}$ Recognition of health behavior change practitioners and using the theory-based approaches regarding to how people behave and what beliefs play roles in encouraging people to perform certain behaviors can help researchers to plan effective interventional programs. ${ }^{12}$ One of the common models in health behavior research is the Health Belief Model (HBM) that has been used in many research studies related to health behaviors. ${ }^{13,14}$ This model investigates individual perceptions and beliefs about health issue based on which individuals must have perceived susceptibility to take preventive actions, then understand the depth of this risk and the seriousness of various physical and mental complications of the problem (perceived severity). If they have a positive analysis on the benefits of preventive behavior (perceived benefits), which perceived severity and benefits also called perceived threat, and the lack of serious barriers (perceived barriers) and receiving the environmental guides (cues to action), and ultimately having an understanding and confidence of adoption of the intended behavior (perceived self-efficacy). ${ }^{12}$ Regarding the determinants influencing the HB vaccine, de Wit and colleagues have investigated the effective role of perceived sensitivity and severity of HB vaccine. ${ }^{11}$ Several studies have also shown that determinants such as low perceived severity, or lack of awareness and lack of belief in the efficacy of the vaccine are effective in declining the vaccine. ${ }^{15-17}$ Van den Dool and co-workers also showed that perceived susceptibility is a strong predictor of the intention to receive an influenza vaccine. ${ }^{18}$ On the other hand, studies have shown that almost $90 \%$ of personnel are aware of $\mathrm{HB}$ vaccination, but only $56.5 \%$ of them completed their vaccination program, and it seems that the current level of HB vaccination is not sufficient to protect healthcare workers against HB. ${ }^{19,20}$

In order to increase vaccination uptake, it seems that determining the predictors affecting the overall performance of HB vaccination in Iranian society is essential and can provide useful findings to health planners. ${ }^{21}$

The aim of our study was to determine the cognitive determinants of HB vaccination uptake among Iranian nurses based on the HBM.

\section{MATERIALS AND METHODS}

\section{Participants and procedure}

This cross-sectional study was conducted among 330 nurses in educational hospitals affiliated to Kermanshah University of Medical Sciences (KUMS), in 2016. The sample size was calculated at 95\% significant level according to the results of a pilot study. The pilot study's finding indicated $29 \%$ of participants reported to have completed (three times) vaccination of $\mathrm{HB}$, so a sample of 330 was estimated.

For appropriate sampling included educational hospitals (Imam Reza, Imam Ali, Imam Khomeini, Farabi, and Taleghani) were considered as the clusters and samples were selected by appropriate assignment of each class according to the number of nurses in each hospital. Coordination with the authorities of KUMS and managers of educational hospitals was done. Using a simple random sampling method, the participants were selected and the questionnaire designed for this purpose was given to them to complete as self-report.

The nurses who worked in Kermanshah educational hospitals were eligible to participate in this study. This 
study has been approved by the institutional review board at the KUMS (KUMS.REC.1395.746).

Of the total 330 nurses, 299 (99.7\%) signed the consent form and voluntarily agreed to participate in this study.

\section{Measures}

The data collection tool included three sections.

\section{Demographic variables}

The first part contained information about five subjects including age (years), sex (male, female), level of education (apprentice, expert, master's degree), work experience (number of years), and history of HB infection among the family or friends (yes, no).

\section{History of $\mathrm{HB}$ vaccination}

The second part included a question regarding the HB vaccination among the participants; "how many times have you received the $\mathrm{HB}$ vaccine (I did not receive it, one, two, and three times)?".

\section{Items of HBM Construct}

The third part included items about the five constructs of HBM. The questionnaire was used by the research team using the patterns. ${ }^{10,11}$ In order to facilitate participants' responses to the items of perceived threat, perceived benefits, perceived barriers, and perceived self-efficacy, all items were standardized to a five-point Likert scale, ranging from 1 (completely disagree) to 5 (completely agree). Furthermore, the response category was designed yes or no for cues to action.

Five items were designed to measure perceived threat from side effects of HB infection (e.g. "HB infection can lead to liver cancer."). Four items were designed to measure perceived benefit of uptake HB vaccine (e.g., "uptake of HB vaccine helps me to prevent HB infection.”). Four items to evaluate perceived barrier to uptake HB vaccine (e.g., "lack of sufficient time to uptake HB vaccine"). Three items were designed for cues to action to uptake HB vaccine (e.g., "Do your family members encourage you to uptake HB vaccine?"). Three items were designed to measure self-efficacy regarding HB vaccination (e.g. “ I am confident I can gather information about HB vaccination."). The Cronbach's alpha for the abovementioned constructs were as follows: perceived threat $(\alpha=0.85)$; perceived barrier $(\alpha=0.82)$, perceived benefit ( $\alpha=0.74)$, perceived self-efficacy $(\alpha=0.78)$, and cues to action $(\alpha=$ 0.65 ) attesting to the internal consistency of the measures.

The content validity was confirmed by an expert group and a questionnaire was used to assess the validity of the questionnaires. A preliminary study was conducted on 30 participants. Using the Cronbach's alpha and split half test, the reliability of the questionnaires was as follows.

\section{Statistical Analysis}

The Statistical Package for the Social Sciences software (SPSS-16) was used for the purpose of data entry, and analysis. Descriptive statistics were used to summarize and organize the data. Bivariate correlations were computed to ascertain the magnitude and direction of the associations between the HBM variables. Backward Wald stepwise multiple logistic regression analysis was performed to predict the HB vaccination on the basis of HBM variables of 1) perceived threat, 2) perceived barrier, 3) perceived benefit, 4) cues to action, and 5) self-efficacy. In addition, stepwise multiple logistic regression analysis was performed to predict the HB vaccination on the basis of background variables. Cronbach's Alpha and split half was used to estimate the internal consistency of the various measures.

\section{RESULTS}

The mean age of the respondents was 30.5 years [SD: 6.62], ranged from 20 to 58 years. In addition, the mean job history of respondents was 79.91 months [95\% CI: 72.29, 87.54], ranged from 2 to 360 months. $34.8 \%(104 / 299)$ of the participates were men, and $65.2 \%(195 / 299)$ were women. Nearly 5\% (15/299), $90 \%(269 / 299)$, and 5\% (15/299) of the respondents reported to be a technician, BSc and MSc, respectively. $8.4 \%(25 / 299)$ of the respondents had positive family or friends history of HB. Furthermore, 58.5\% (175/299) of the participants reported to have completed (three times) vaccination against $\mathrm{HB}$. In addition, 45.2\% (47/104) and $65.6 \%(128 / 195)$ of men and women reported to have completed (three times) vaccination against $\mathrm{HB}$, respectively.

Table 1 shows the Zero-order correlations. Significance levels at the 0.01 and 0.05 were the criteria for the analysis. The bivariate assessment of variables revealed that there were signs of multicollinearity among HBM 
Table 1: Correlation between different components of HB

\begin{tabular}{lcccccc}
\hline Components & Mean (SD) & Range & X1 & X2 & X3 & X4 \\
\hline X1. Perceived threat & $19.58(3.88)$ & $5-25$ & 1 & & & \\
\hline X2. Perceived benefit & $15.82(2.66)$ & $4-20$ & $0.208^{* *}$ & 1 & & \\
\hline X3. Perceived barrier & $13.23(3.66)$ & $4-20$ & $-0.130^{*}$ & $-0.132^{*}$ & 1 & \\
\hline X4. Perceived self-efficacy & $12.26(1.99)$ & $3-15$ & $0.369^{* *}$ & $0.333^{* *}$ & -0.047 & 1 \\
\hline X5. Cues to action & $1.95(0.96)$ & $0-3$ & $0.173^{* *}$ & $0.162^{* *}$ & -0.091 & $0.149^{*}$ \\
\hline
\end{tabular}

* Correlation is significant at the 0.05 level (2-tailed)

** Correlation is significant at the 0.01 level (2-tailed)

Table 2: Associations between HBM components and HB vaccination uptake

\begin{tabular}{|c|c|c|c|c|}
\hline Components & Vaccination uptake & Mean (SD) & $\mathbf{t}$ & Sig \\
\hline \multirow[t]{2}{*}{ Perceived threat } & Not Fully & $17.65(4.60)$ & -7.955 & $<0.001$ \\
\hline & Fully & $20.94(2.50)$ & & \\
\hline \multirow[t]{2}{*}{ Perceived benefit } & Not Fully & $15.49(2.72)$ & -1.835 & 0.068 \\
\hline & Fully & $16.06(2.60)$ & & \\
\hline \multirow[t]{2}{*}{ Perceived barrier } & Not Fully & $13.58(3.37)$ & 1.378 & 0.169 \\
\hline & Fully & $12.98(3.84)$ & & \\
\hline \multirow[t]{2}{*}{ Perceived self-efficacy } & Not Fully & $11.60(2.19)$ & -4.996 & $<0.001$ \\
\hline & Fully & $12.73(1.69)$ & & \\
\hline \multirow[t]{2}{*}{ Cues to action } & Not Fully & $1.73(1.11)$ & -3.180 & 0.002 \\
\hline & Fully & $2.10(0.81)$ & & \\
\hline
\end{tabular}

Table 3: Multiple logistic regression analysis for socio-demographic characteristics related to complete (three times) vaccination of HB (final model. Step 4)

\begin{tabular}{|c|c|c|c|c|c|}
\hline Variables & & Crude OR (95\% CI) & $P$ value & Adjusted OR (95\% CI) & $P$ value \\
\hline \multirow[t]{2}{*}{ Sex } & Male & 1 & - & 1 & - \\
\hline & Female & $2.31(1.42-3.76)$ & 0.001 & $2.50(1.52-4.12)$ & $<0.001$ \\
\hline \multirow{2}{*}{$\begin{array}{l}\text { Positive family or } \\
\text { friends history of } \mathrm{HB}\end{array}$} & No & 1 & - & 1 & - \\
\hline & Yes & $3.07(1.12-8.42)$ & 0.029 & $3.70(1.31-10.42)$ & 0.013 \\
\hline
\end{tabular}

variables. The findings indicate that for the sample, perceived threat was significantly correlated to self-efficacy $(r=0.369)$, benefit $(r=0.208)$, and cues to action $(r=0.173)$, while inversely correlated with barrier $(r$ $=-0.130$ ). Self-efficacy was significantly correlated to benefit $(r=0.333)$, and cues to action $(r=0.149)$, but there was no significant correlation between self-efficacy and barrier $(\mathrm{r}=-0.047)$. Barrier was significantly inversely correlated to benefit $(r=-0.132)$, also there was no significant correlation between barrier and cues to action $(r=-0.091)$. Benefit was significantly related to cues to action $(\mathrm{r}=0.162)$.

As can be seen in table 2, means of perceived threat, perceived self-efficacy, and cues to action among nurses who are fully vaccinated were significantly higher than those who are not. Furthermore, there were no significantly associations between perceived benefit and perceived barrier with $\mathrm{HB}$ vaccination uptake.

Logistic regression (backward stepwise method) building procedure was conducted and finally on 4th step the procedure was stopped and the best model was selected. Among the socio-demographic determinants, female sex with adjusted odds ratio estimate of 2.507 [95\% CI: $1.523-4.125]$ and positive family or friends history of HB with odds ratio estimate of 3.706 [95\% CI: 1.317-10.425] were the most influential predictive determinants for doing complete (three times) HB vaccination (table 3).

As mentioned in statistical analyses, a step-wise model building procedure was conducted and finally on 
Table 4: Multiple logistic regression analysis for HBM variables related to doing complete (three times) vaccination of $\mathrm{HB}$ (final model. Step 3)

\begin{tabular}{|c|c|c|c|c|c|c|}
\hline \multirow{2}{*}{ Components } & \multirow{2}{*}{ B } & \multirow{2}{*}{ S.E. } & \multirow{2}{*}{ Odds Ratio } & \multicolumn{2}{|c|}{ 95\% Confidence intervals } & \multirow{2}{*}{$P$ value } \\
\hline & & & & Lower & Upper & \\
\hline Perceived threat & 0.234 & 0.044 & 1.26 & 1.16 & 1.37 & $<0.001$ \\
\hline Perceived self-efficacy & 0.165 & 0.074 & 1.17 & 1.02 & 1.36 & 0.026 \\
\hline Cues to action & 0.289 & 0.140 & 1.33 & 1.01 & 1.75 & 0.039 \\
\hline
\end{tabular}

3rd step the procedure stopped and the best model was selected. Among the HBM variables perceived threat with odds ratio estimate of 1.264 [95\% CI: 1.160-1.376], perceived self-efficacy with odds ratio estimate of 1.179 [95\% CI: 1.020-1.363], and cues to action with odds ratio estimate of 1.335 [95\% CI: 1.015-1.756] are the most influential predictors of doing complete (three times) vaccination of $\mathrm{HB}$.

\section{DISCUSSION}

The aim of this study was to determine the predictive determinants for performing a complete vaccination of HB among nurses in educational hospitals in Kermanshah, affiliated to KUMS. The findings of the study showed that $58.5 \%$ of the participants had a history of $\mathrm{HB}$ vaccination. This finding is largely in accordance with the findings of Kisic-Tepavcevic and colleagues among healthcare providers in Serbia, who reported an acceptance rate of HB vaccination $(66.2 \%){ }^{22}$ Tibdewal and colleagues also reported that among medical and dental students in India, $55.9 \%$ of them completed the immunization program against $\mathrm{HB}^{23}$ Since the nurses are a member of the care team and a large part of the medical community, and given the wide range of care and professional roles in different departments of hospitals, the risk of occupational injury is higher and it is expected that they have better preventive behaviors. But our findings indicated that $41.5 \%$ of them had not fully vaccinated against HB, which could be a warning sign to health policy makers in Iran, and the need for more attention to this issue and the identification of factors determining behaviors for planning the training programs more precisely.

Our findings showed that sex was a predictor of receiving the $\mathrm{HB}$ vaccine as the proportion of receiving $\mathrm{HB}$ vaccination was higher among women. In this regard, sex was introduced as one of the social determinants of health. Sexual roles and norms affect the access of women and men to health services and how health systems respond to their different needs. ${ }^{24}$ On the contrary, the findings of this study have shown in several studies that women have an unfavorable situation in terms of access to health services. ${ }^{25-27}$ In this regards Gonzales found women more willing than men to seek out HB information, which may explain women's higher rates of immunization. ${ }^{28,29}$ In addition, it should be noted that Iran is one of the countries with a high level of attention to the health of women, and there are many programs regarding the provision of women's health services in Iran. ${ }^{30}$ It seems that in educations related to promotion of health services in Iran, special attention should be paid to men.

Other finding of the present study is the more chance of nurses who have had a history of becoming acquainted with a patient with $\mathrm{HB}$ to receive $\mathrm{HB}$ vaccination. In many cases, screening and health promoting behaviors have been reported among people living with a patient in their family or friends. ${ }^{31,32}$ They are likely to have higher sensitivity, and hence have more preventative behaviors.

The perceived threat, perceived self-efficacy, and guidance for action were the most important predictors of $\mathrm{HB}$ vaccination. In this regard, the study by Grace in Vietnamese immigrants also showed a high perceived sensitivity to $\mathrm{HB}$ and vaccination against it. ${ }^{33}$ In the study by Youafzai and colleagues, which focused on health care workers in the Pakistani suburbs, perceived severity structures were more important and a stronger predictor of needle stick prophylaxis. ${ }^{34}$ De Wit and others in their study investigated the cognitive determinants affecting the $\mathrm{HB}$ vaccination among men who had sex with men using the HBM and the theory of plan behavior. They reported in their study that men who received uptake a HB vaccine had higher significantly perceived risk of HB. ${ }^{11}$ Brewer and colleagues also showed that susceptibility and severity were predictors of receiving vaccination in their meta-analysis. ${ }^{35}$ It seems that due to eliminating the 
perceived benefits and barriers of the model in anticipation of receiving $\mathrm{HB}$ vaccination, may be the familiarity of nurses with regard to HB disease and strategies for coping with perceived barriers, and therefore fewer barriers to adopting prevention behaviors. According to the findings of this study, it seems that the more use of external action guides in promoting the threat and perceived self-efficacy of nurses, in order to increase the probability of decision making for the preventive behavior of HB disease; service managers of nursing and education officials in hospitals should pay attention to these determinants during the training programs, then they can expect to be proactive in preventing $\mathrm{HB}$ disease.

This study had a few limitations. First, the study was retrospective in nature and relied heavily on recollection of past events. Second, the information is based on selfreporting, which always faces the risk of recall bias and we do not know how it could have affected the results. Third, the internal consistency of the questionnaire was relatively low $(\alpha=0.65)$ for assessing cues to action. Finally, the high rejection rate is another limitation of our study.

The findings of this study suggest the following for the development of vaccination promotion programs: given the lower uptake of $\mathrm{HB}$ vaccination among male nurses, more attention needs to be paid to their vaccination uptake. The authors suggest the use of fear appeal behavioral change methods with emphasizing the promotion of self-efficacy, and in the interpersonal using of descriptive and perceptual norms behavioral change methods.

\section{ACKNOWLEDGEMENTS}

This article is a part of research project supported by KUMS, Kermanshah, Iran. We would like to thank deputy of research of KUMS for financial support of this study.

\section{ETHICAL APPROVAL}

There is nothing to be declared.

\section{CONFLICT OF INTEREST}

The authors declare no conflict of interest related to this work.

\section{REFERENCES}

1. Locarnini S, Hatzakis A, Chen DS, Lok A. Strategies to control hepatitis B: Public policy, epidemiology, vaccine and drugs. J Hepatol 2015;62:S76-86.

2. Poorolajal J, Majdzadeh R. Prevalence of chronic hepatitis B infection in Iran: a review article. $J$ Res Med Sci 2009;14:249-58.

3. Filippelli M, Lionetti E, Gennaro A, Lanzafame A, Arrigo T, Salpietro C, et al. Hepatitis B vaccine by intradermal route in non-responder patients: an update. World $J$ Gastroenterol 2014;20:10383-94. doi:10.3748/wjg.v20. i30.10383.

4. Mohammadi Z, Keshtkar A, Eghtesad S, Jeddian A, Pourfatholah AA, Maghsudlu M, et al. Epidemiological profile of hepatitis B virus infection in Iran in the past 25 years; a systematic review and meta-analysis of general population studies. Middle East J Dig Dis 2016;8:5-18. doi:10.15171/mejdd.2016.01.

5. Schweitzer A, Horn J, Mikolajczyk RT, Krause G, Ott JJ. Estimations of worldwide prevalence of chronic hepatitis $\mathrm{B}$ virus infection: a systematic review of data published between 1965 and 2013. The Lancet 2015;386:1546-55. doi:10.1016/S0140-6736(15)61412-X.

6. Hahné SJ, Veldhuijzen IK, Wiessing L, Lim TA, Salminen $M$, van de Laar M. Infection with hepatitis $B$ and $C$ virus in Europe: a systematic review of prevalence and costeffectiveness of screening. BMC Infect Dis 2013;13:181. doi:10.1186/1471-2334-13-181.

7. Kabir A, Pazouki A, Jafari M, Mokhber S, Vaziri M, Alavian SM. Comparing Anti-hepatitis B Antibody Level in Iranian Obese or Overweight with Non-obese Cases. Iran Biomed J 2017;21:197-202. doi:10.18869/acadpub. ibj.21.3.197

8. Sayehmiri K, Azami M, Nikpey S, Borji M, Sayehmiri F. Hepatitis B vaccination coverage in health personnel of Iran: a systematic review and meta-analysis study. Iran $J$ Epidemiol 2015;11:1-10.

9. Lehmann BA, Ruiter RA, Van Dam D, Wicker S, Kok G. Sociocognitive predictors of the intention of healthcare workers to receive the influenza vaccine in Belgian, Dutch and German hospital settings. $J$ Hosp Infect 2015;89:202-9. doi:10.1016/j.jhin.2014.11.009.

10. Lehmann BA, Chapman GB, Franssen FM, Kok G, Ruiter RA. Changing the default to promote influenza vaccination among health care workers. Vaccine 2016;34:138992. doi:10.1016/j.vaccine.2016.01.046.

11. de Wit JB, Vet R, Schutten M, van Steenbergen J. Socialcognitive determinants of vaccination behavior against hepatitis B: an assessment among men who have sex with men. Prev Med 2005;40:795-802. doi:10.1016/j. ypmed.2004.09.026.

12. Mirzaei-Alavijeh M, Kok G, Niknami S, Motlagh ME. Family-based cognitive factors effective on preventing the onset of substance use in iranian society's children: applying the intervention mapping protocol. Acta Medica Mediterranea 2016;32:1015-20. 
13. Baghiani-Moghadam M, Mirzaei-Alavijeh M, Zolghadr R. Knowledge, risk perceptions and behavioral intentions among elementary school teachers of Yazd regarding hepatitis A. Govaresh 2012;17:84-90.

14. Jalilian F, Hazavehei SM, Vahidinia AA, Jalilian M, Moghimbeigi A. Prevalence and related factors for choosing self-medication among pharmacies visitors based on Health Belief Model in Hamadan Province, West of Iran. J Res Health Sci 2013;13:81-5.

15. Cohen DL, John Casken RN. Why are healthcare workers so resistant to the acceptance of influenza vaccine? A review of the literature to examine factors that influence vaccine acceptance. International Journal of Caring Sciences 2012;5:26-35.

16. del Carmen Aguilar-Díaz F, Jiménez-Corona ME, Poncede-León-Rosales S. Influenza vaccine and healthcare workers. Arch Med Res 2011;42:652-7. doi:10.1016/j. arcmed.2011.12.006.

17. Hollmeyer HG, Hayden F, Poland G, Buchholz U. Influenza vaccination of health care workers in hospitals - a review of studies on attitudes and predictors. Vaccine 2009;27:3935-44. doi:10.1016/j.vaccine.2009.03.056.

18. Van den Dool C, Van Strien AM, Looijmans-Van Den Akker I, Bonten MJ, Sanders EA, Hak E. Attitude of Dutch hospital personnel towards influenza vaccination. Vaccine 2008;26:1297-302. doi:10.1016/j. vaccine. 2007.12.045.

19. Maltezou HC, Gargalianos P, Nikolaidis P, Katerelos P, Tedoma N, Maltezos E, et al. Attitudes towards mandatory vaccination and vaccination coverage against vaccinepreventable diseases among health-care workers in tertiary care hospitals. $J$ Infect 2012;64:319-24. doi:10.1016/j. jinf.2011.12.004.

20. Mahboobi N, Agha-Hosseini F, Mahboobi N, Safari S, Lavanchy D, Alavian SM. Hepatitis B virus infection in dentistry: a forgotten topic. J Viral Hepat 2010;17:30716. doi:10.1111/j.1365-2893.2010.01284.x.

21. Morowatishaifabad MA, Zare Sakhvidi MJ, Gholianavval M, Masoudi Boroujeni D, Alavijeh MM. Predictors of hepatitis B preventive behavioral intentions in healthcare workers. Saf Health Work 2015;6:139-42. doi:10.1016/j. shaw.2014.12.001.

22. Kisic-Tepavcevic D, Kanazir M, Gazibara T, Maric G, Makismovic N, Loncarevic G, et al. Predictors of hepatitis $B$ vaccination status in healthcare workers in Belgrade, Serbia, December 2015. Euro Surveill 2017;22:30515. doi:10.2807/1560-7917.ES.2017.22.16.30515.

23. Tibdewal H, Barad P, Kumar S. Comparing dental and students' knowledge and attitudes toward HepatitisB and $\mathrm{C}$ and HIV infected patients in India. J International Oral Health 2009;1:20-30.

24. Regitz-Zagrosek V. Sex and gender differences in health. EMBO Rep 2012;13:596-603. doi:10.1038/embor.2012.87.

25. Regitz-Zagrosek V. Therapeutic implications of the gen- der-specific aspects of cardiovascular disease. Nat Rev Drug Discov 2006;5:425-38. doi:10.1038/nrd2032.

26. Regitz-Zagrosek V, Petrov G, Lehmkuhl E, Smits JM, Babitsch B, Brunhuber C, et al. Heart transplantation in women with dilated cardiomyopathy. Transplantation 2010;89:236-44. doi:10.1097/TP.0b013e3181c35255.

27. Humphries KH, Kerr CR, Connolly SJ, Klein G, Boone JA, Green M, et al. New-onset atrial fibrillation: sex differences in presentation, treatment, and outcome. $\mathrm{Circu}$ lation 2001;103:2365-70.

28. Gonzales RM, Glik DC, Prelip M, Bourque L, Yuen J, Ang A, et al. Risk perceptions and behavioral intentions for Hepatitis B: how do young adults fare? Health Educ Res 2006;21:654-61. doi:10.1093/her/cyl047.

29. Middleman AB. Race/ethnicity and gender disparities in the utilization of a school-based hepatitis B immunization initiative. J Adolesc Health 2004;34:414-9. doi: 10.1016/j.jadohealth.2003.08.009.

30. Mirzaei-Alavijeh M, Motlagh MI, Jalilian F, Allameh M, Karami-Matin B, Firoozabadi A, Mahboubi M. Utilization of Iranian Women Health Services (SABA) in the West of Iran: An Application of Social Cognitive Theory. Res J Med Sci 2016;10;429-33.

31. Sim HL, Seah M, Tan SM. Breast cancer knowledge and screening practices: a survey of 1000 Asian women. Singapore Med J 2009;50:132-8.

32. Çam $\mathrm{O}$, Gümüs $\mathrm{AB}$. Breast cancer screening behavior in Turkish women: Relationships with health beliefs and self-esteem, body perception and hopelessness. Asian Pac J Cancer Prev 2009;10:49-56.

33. Ma GX, Fang CY, Shive SE, Toubbeh J, Tan Y, Siu P. Risk perceptions and barriers to Hepatitis B screening and vaccination among Vietnamese Immigrants. J Immigr Minor Health 2007;9:213-20. doi:10.1007/s10903-006-9028-4.

34. Yousafzai MT, Siddiqui AR, Janjua NZ. Health belief model to predict sharps injuries among health care workers at first level care facilities in rural Pakistan. Am J Ind Med 2013;56:479-87. doi:10.1002/ajim.22117.

35. Brewer NT, Chapman GB, Gibbons FX, Gerrard M, McCaul KD, Weinstein ND. Meta-analysis of the relationship between risk perception and health behavior: the example of vaccination. Health Psychol 2007;26:136-45. doi:10.1037/0278-6133.26.2.136. 